\title{
Tailored mesh generation for NEFEM
}

\author{
Xi Zou, Ruben Sevilla, Oubay Hassan and Kenneth Morgan \\ Zienkiewicz Centre for Computational Engineering \\ College of Engineering, Swansea University \\ Swansea SA1 8EN, Wales, UK \\ e-mail: \{xi.zou, r.sevilla, o.hassan, k.morgan\}@swansea.ac.uk
}

\begin{abstract}
Preparing geometric models suitable for engineering simulations is known to be a major bottleneck when dealing with complex geometries because an excessive number of human hours are required for the information transfer from a computer aided design (CAD) model to a proper computer aided engineering (CAE) model. CAD models frequently involve a level of detail much greater than that required to perform a numerical simulation. Over the last two decades, a large effort has been placed on the research of algorithms for the de-featuring of complex CAD models. However, de-featuring cannot be fully automatised. Firstly, it is usually not possible to know, a priori, the effect of de-featuring on the results of a simulation that could involve mesh size dependency. Secondly, de-featuring is often physics-dependent, i.e., mesh requirements are different for fluid dynamics, electromagnetics, acoustics, heat transfer or structural mechanics applications. Finally, de-featuring is dependent upon the level of approximation required.

When small geometric features are present in a CAD model, traditional mesh generators [1] will produce small, often distorted, elements, when the spacing specified by the user is much larger than the size of the small features.

The NURBS-enhanced finite element method (NEFEM) [2] addresses this problem by completely decoupling the concepts of geometric and solution approximation. These two concepts are tightly coupled in the large majority of available solvers through the isoparametric mapping between a well-shaped reference element and the deformed geometric-fitting elements. With NEFEM, the geometric description of the boundary uses the boundary representation (B-rep) immediately available in the CAD model, whereas the approximation of the solution still uses polynomial functions [3]. As a result, the error due to geometric approximation is completely removed. This new concept completely avoids the need for de-featuring and, at the same time, can use meshes where the size of elements is entirely decided by the user and is no longer limited by the presence of geometric features smaller than the specified spacing.

This work will present a new tailored mesh generation technique [4] where boundary faces span across multiple surfaces and retain the exact B-rep. The surface mesh generation process will be detailed, including the new data structures that have been devised to store the information required by NEFEM elements. New algorithms, in particular, the local enhanced advancing front method, have been implemented to fulfil the creation of boundary faces that span across multiple surfaces. The creation of the volume mesh will be outlined, and several examples will be presented to show the potential of the proposed technique.
\end{abstract}




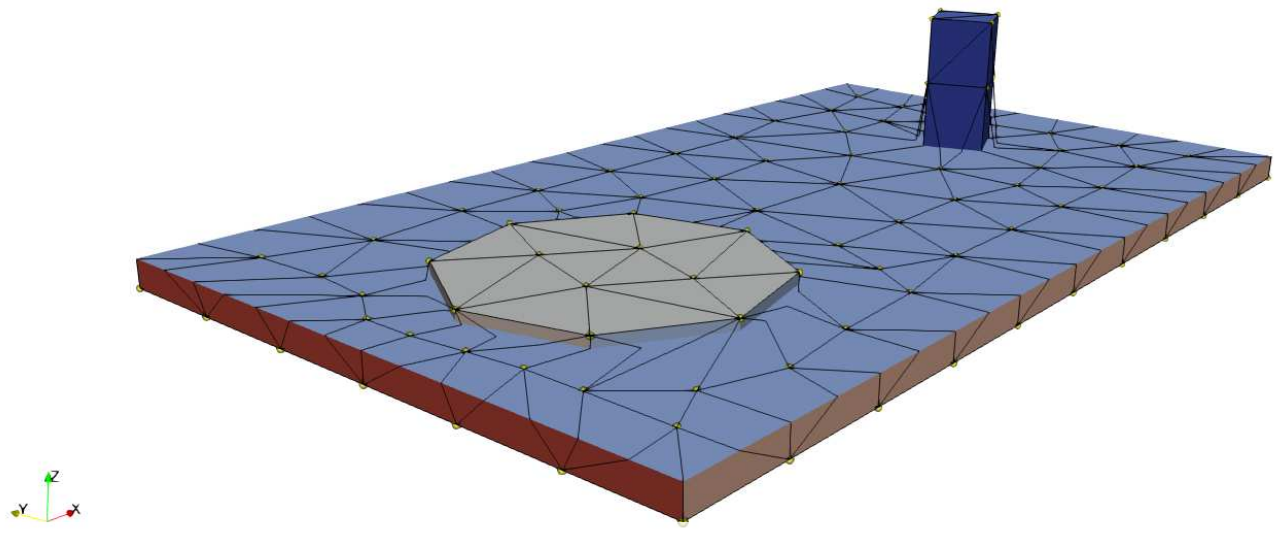

Figure 1: A NEFEM surface mesh.

\section{REFERENCES}

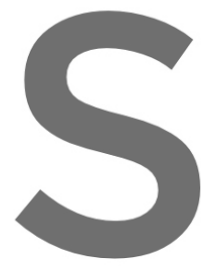

[1] Xie ZQ, Sevilla R, Ha for $3 \mathrm{D}$ finite element

[2] Sevilla R, Fernández-ring Int. J. Num, Meth.
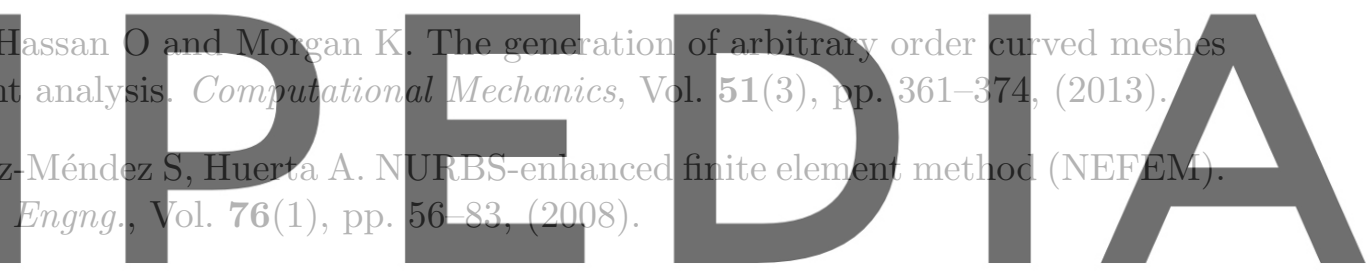

[3] Sevilla R, Fernández-Méndez S, Huerta A. Comparison of high-order curved finite elements.

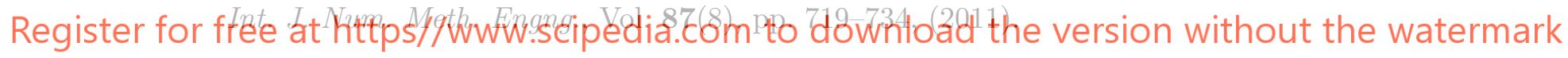

[4] Sevilla R, Rees L and Hassan O. The generation of triangular meshes for NURBS-enhanced FEM. Int. J. Num. Meth. Engng., Vol. 108, pp. 941-968, (2016). 